\title{
Register in Versorgungsforschung und Qualitätssicherung
}

\author{
Nicole Stecka, Stefanie Hostettlerb, Esther Kraft ${ }^{\mathrm{b}}$, Claudia Berlin ${ }^{\mathrm{a}}$, Adrian Spörria, Marcel Zwahlen ${ }^{\mathrm{a}}$ \\ ${ }^{a}$ Institut für Sozial- und Präventivmedizin der Universität Bern; ${ }^{b} \mathrm{FMH}$, Abteilung Daten, Demographie und Qualität
}

Die Zahl der medizinischen Register in der Schweiz nimmt zu. Die krankheits- oder behandlungsspezifischen Register werden entweder auf freiwilliger oder obligatorischer Basis geführt und dienen verschiedenen Zwecken. Sie sind für Qualitätssicherung und (Versorgungs-)Forschung ein wichtiger Pfeiler, aber nicht alle drängenden Fragen können damit beantwortet werden. Informationen aus Registern werden vermehrt auch bei Health Technology Assessments einbezogen. Der Nutzen steht und fällt mit der Qualität der Register, diese misst sich an Abdeckung und Repräsentativität, Vollständigkeit und Korrektheit der Angaben, insbesondere auch, ob Informationen über den Verlauf nach Diagnose und initialer Behandlung zuverlässig erfasst werden.

Rund 100 medizinische Register sind derzeit auf der Online-Plattform «Forum medizinische Register Schweiz» der FMH (www.fmh.ch/services/qualitaet/ forum_medizinische_register.html) verzeichnet [1]. Die Anzahl der dokumentierten Register ist mit jeder Aktualisierung weiter angestiegen. Die Bandbreite der Register reicht von A wie AMDS - das Absolute Mini-

\section{Online-Plattform}

"Forum medizinische Register Schweiz»

Seit 2012 dokumentiert die Schweizerische Akademie für Qualität in der Medizin SAOM medizinische Register auf ihrer Website [1]. Diese Plattform zeigt die Bandbreite der medizinischen Register auf, und mit der Suchmaschine findet man mit wenigen Klicks das gesuchte Register.

Die Online-Plattform hat zum Ziel, Interessierten die gesammelten Informationen über die dokumentierten Register zugänglich zu machen, die Vernetzung unter den Registerbetreibenden zu fördern und als Orientierungshilfe beim Aufbau von neuen Registern zu dienen. Sie enthält für jedes Register einheitlich strukturierte Informationen, bestehend aus einem Kurzbeschrieb und Angaben zur geographischen Abdeckung, Datenarten und -erfassung, Population, Teilnahmemöglichkeit, Datenzugang, Finanzierung oder Berichterstattung.

Die Online-Plattform wird durch die SAOM regelmässig aktualisiert und dient ihr als Basis für weitere Arbeiten zum Thema Register.

www.fmh.ch $\rightarrow$ Qualität $\rightarrow$ Forum medizinische Register mal Data Set über die Tätigkeit im Bereich Anästhesiologie - bis zu Z wie ZDR, dem BAG-Register der Dosen, die von den beruflich strahlenexponierten Personen in der Schweiz akkumuliert werden. Was aber ist ein medizinisches Register, wozu wird es gebraucht und welche Anforderungen muss es erfüllen? Etwa so unterschiedlich wie die Register selbst sind auch die in der Literatur vorhandenen Definitionen eines medizinischen Registers. Als Konsens sprechen sie «von einer systematischen Sammlung von einheitlichen Daten innerhalb eines definierten Untersuchungskollektivs» $[2,3]$. Weiter geht ein europäisches Dokument von 2015 [4]: «Register sind ein organisiertes System, um Daten und Informationen von einer Gruppe von Menschen zu sammeln, zu analysieren und zu verbreiten, die durch eine bestimmte Erkrankung, einen $\mathrm{Zu}$ stand, eine Exposition oder eine medizinische Leistung definiert ist; und sie dienen zum Voraus bestimmten wissenschaftlichen, klinischen und/oder politischen Zwecken.»

Dass sich die Definitionen teilweise stark unterscheiden und verschieden detailliert ausfallen, liegt auch an den unterschiedlichen Ansprüchen an verschiedene Register. Einige - etwa im Bereich der Infektionskrankheiten - dienen sowohl als Frühwarnsystem wie auch um längerfristig die Kontrollmassnahmen zu evaluieren, andere zu Forschungszwecken oder zur Herstel- 
lung von Transparenz. So müssen in der Schweiz über 50 verschiedene Erkrankungen dem Bundesamt für Gesundheit gemeldet werden, zum Beispiel HIV, Malaria, Masern, Tuberkulose und ab 2020 Krebserkrankungen. Der Grossteil der Register ist freiwillig. Häufig

\section{Über 50 verschiedene Erkrankungen müssen dem Bundesamt für Gesundheit gemeldet werden, zum Beispiel HIV, Malaria, Masern, Tuberkulose.}

geht die Initiative von Fachärzten beziehungsweise von den medizinischen Fachgesellschaften, in Einzelfällen aber auch von Patientengruppen aus. Um die einzelnen Registerformen besser voneinander abgrenzen zu können, wird in der Literatur eine Kategorisie- rung zum Beispiel in Public-Health-, Medizinische, Epidemiologische, Klinische und PatientInnen-Register vorgeschlagen [2, 3, 5, 6] (Tab. 1). Allerdings ist die Abgrenzung der verschiedenen Registertypen - und teilweise auch von Kohorten - nicht immer eindeutig, und ein Register kann verschiedenen Zwecken dienen.

\section{Daten sammeln, aber richtig}

Unabhängig vom Zweck eines Registers müssen gewisse Qualitätskriterien erfüllt werden. Nur so kann der mit dem Aufbau und Betrieb eines Registers verbundene hohe organisatorische, zeitliche und finanzielle Aufwand gerechtfertigt werden [7, 8]. Ein unsorgfältig oder gar schlecht geführtes Register ist im

Tabelle 1: Mögliche Kategorisierung verschiedener Registertypen.

\begin{tabular}{|c|c|c|c|c|}
\hline Art des Registers & Einschlusskriterien & Resultate & Was wird womit verglichen? & $\begin{array}{l}\text { Beispiele von möglichen } \\
\text { Aussagen }\end{array}$ \\
\hline $\begin{array}{l}\text { Public-Health-Register } \\
\text { Beispiele: } \\
\text { Register der Personen nach } \\
\text { verabreichten Impfungen } \\
\text { Register gescreenter Personen }\end{array}$ & $\begin{array}{l}\text { Region/Teilnahme } \\
\text { an Impf- oder Scree- } \\
\text { ning-Programm }\end{array}$ & $\begin{array}{l}\text { Abdeckung des } \\
\text { Programms in der } \\
\text { Zielbevölkerung } \\
\text { Nebenwirkungen nach } \\
\text { Impfungen }\end{array}$ & $\begin{array}{l}\text { Vergleich der Häufigkeit von } \\
\text { Nebenwirkungen nach Art des } \\
\text { Impfstoffes } \\
\text { Vergleich von Folgeabklärun- } \\
\text { gen nach Brustkrebsscreening } \\
\text { nach Regionen }\end{array}$ & $\begin{array}{l}\text { Abdeckung des Programms } \\
\text { ist tief sowohl bei jüngeren als } \\
\text { auch bei älteren Personen } \\
\text { Inzidenz von Nebenwirkungen } \\
\text { nach trivalentem Impfstoff ist } \\
\text { nicht unterschiedlich von der } \\
\text { Inzidenz nach bivalentem } \\
\text { Impfstoff }\end{array}$ \\
\hline $\begin{array}{l}\text { Medizinische Register } \\
\text { Beispiele: } \\
\text { Register der Patienten mit } \\
\text { Prothesenoperation } \\
\text { Register der Patienten mit } \\
\text { einem herzchirurgischen } \\
\text { Eingriff }\end{array}$ & $\begin{array}{l}\text { Bestimmte Erkran- } \\
\text { kung oder medizini- } \\
\text { scher Eingriff/ } \\
\text { Behandlung }\end{array}$ & Verlauf, Komplikationen & $\begin{array}{l}\text { Vergleich Behandlung A mit } \\
\text { Behandlung B beziehungs- } \\
\text { weise mit Patientlnnen ohne } \\
\text { Behandlung } \\
\text { Vergleich verschiedener } \\
\text { Spitäler }\end{array}$ & $\begin{array}{l}\text { Revisionslast ist höher bei } \\
\text { Implantat A als bei Implantat B } \\
\text { Sterblichkeit nach Herzchirur- } \\
\text { gie ist in einer Klinik höher als } \\
\text { in anderen Kliniken korrigiert } \\
\text { für Risikofaktoren wie den } \\
\text { Euroscore II }\end{array}$ \\
\hline $\begin{array}{l}\text { Epidemiologisches Register } \\
\text { Beispiele: } \\
\text { Register aller Todesfälle } \\
\text { mit Todesursachen } \\
\text { Register der Personen } \\
\text { mit Krebsdiagnosen }\end{array}$ & Zeitpunkt/Region & Inzidenz, Mortalität & $\begin{array}{l}\text { Vergleich mit anderen } \\
\text { Zeitpunkten } \\
\text { Vergleich von Regionen }\end{array}$ & $\begin{array}{l}\text { Rückgang von tödlichen } \\
\text { Myokardinfarkten im Vergleich } \\
\text { zum Vorjahr } \\
\text { Die altersstandardisierte } \\
\text { Brustkrebsinzidenz bei Frauen } \\
\text { unter } 40 \text { nimmt zu }\end{array}$ \\
\hline $\begin{array}{l}\text { Klinische Register } \\
\text { Beispiele: } \\
\text { PatientInnen eines Spitals mit } \\
\text { einer Brustkrebsdiagnose } \\
\text { Register der Transplantations- } \\
\text { empfängerInnen }\end{array}$ & $\begin{array}{l}\text { Behandelndes } \\
\text { Krankenhaus }\end{array}$ & $\begin{array}{l}\text { Verlauf nach Behand- } \\
\text { lung, Wechsel oder Ab- } \\
\text { bruch der Behandlung }\end{array}$ & $\begin{array}{l}\text { Daten über den Verlauf von } \\
\text { definierten Patientengruppen, } \\
\text { Verlauf, Versorgungsalltag }\end{array}$ & $\begin{array}{l}\text { 5-Jahres-Überleben nach } \\
\text { Stadium der Erkrankung } \\
\text { bei Diagnose ist gleich wie } \\
\text { vor } 10 \text { Jahren } \\
\text { Die Häufigkeit des Behand- } \\
\text { lungswechsels ist höher bei } \\
\text { höherem Stadium der Krank- } \\
\text { heit }\end{array}$ \\
\hline $\begin{array}{l}\text { Patientinnen-Register } \\
\text { (zum Teil auch Kohorten) } \\
\text { Beispiele: } \\
\text { Register von Personen mit MS } \\
\text { Register von Personen mit } \\
\text { neuromuskulärer Erkrankung }\end{array}$ & $\begin{array}{l}\text { Bestimmte } \\
\text { Erkrankung }\end{array}$ & $\begin{array}{l}\text { Verlauf, erhaltene } \\
\text { Behandlungen, Lebens- } \\
\text { qualität }\end{array}$ & $\begin{array}{l}\text { Vergleiche des Verlaufs unter } \\
\text { verschiedenen Behandlungen, } \\
\text { allenfalls verschiedenen gene- } \\
\text { tischen Varianten der Erkran- } \\
\text { kung }\end{array}$ & $\begin{array}{l}\text { Der Anteil der Personen mit } \\
\text { MS mit beruflicher Tätigkeit } \\
\text { nimmt zu } \\
\text { Ein Teil der Lähmungen bei } \\
\text { Menschen mit Rückenmark- } \\
\text { verletzungen ist reversibel }\end{array}$ \\
\hline
\end{tabular}


schlimmsten Fall nicht nur nutzlos, sondern kann gar zu unzuverlässigen Informationen und falschen Schlüssen oder Entscheidungen führen.

Ein wichtiger Anspruch an ein Register ist Kontinuität. Das Register muss so aufgebaut sein, dass die Führung im Alltag realistisch ist, auch wenn die allfällige Anfangseuphorie nachgelassen hat. Die Finanzierung sollte möglichst langfristig gesichert sein, ebenso die Teilnahme der Stellen, welche die Information liefern müssen.

Ein weiteres zentrales Kriterium für die Qualität eines Registers ist die Vollständigkeit. Diese betrifft meh-

\section{Das Register muss so aufgebaut sein, dass die} Führung im Alltag realistisch ist, auch wenn die allfällige Anfangseuphorie nachgelassen hat.

rere Dimensionen. Einerseits müssen möglichst alle den Anforderungen entsprechenden Fälle registriert werden beziehungsweise die Möglichkeit haben, registriert zu werden. Das heisst, wenn eine Klinik ein Register über sämtliche Infektionen führt, darf nicht in einer Abteilung jeder Fall dokumentiert werden, während in einer Abteilung die Infektionen nur registriert werden, wenn jemand gerade Zeit hat. Dass die Meldung an ein Register obligatorisch ist, heisst noch lange nicht, dass es vollständig ist. So geht man etwa bei den ebenfalls meldepflichtigen Chlamydien davon aus, dass die Zahl der gemeldeten Fälle nur einen Bruchteil der oftmals asymptomatisch verlaufenden Erkrankungen abdecken. Andererseits müssen aber auch pro Fall alle Informationen gleich detailliert registriert werden. Meldet zum Beispiel eine Klinik zwar alle Fälle, aber nicht alle Komplikationen, führt dies zu

\section{Pro Fall müssen alle Informationen gleich detailliert registriert werden.}

falschen Schlüssen. Dasselbe ist der Fall, wenn etwa Komplikationen nach einem Eingriff registriert werden, nicht aber Komplikationen bei Patientinnen und Patienten im Register, bei denen auf den Eingriff verzichtet wurde.

Wichtig und anspruchsvoll ist weiter die Konsistenz der Daten. Klare Richtlinien müssen eine möglichst einheitliche Registrierung gewährleisten, unabhängig von der meldenden Person. Wenn immer möglich sollten diese auf nationale oder internationale Standards gestützt werden. Dies ist insbesondere dann von Bedeutung, wenn die zu erhebende Information einen Interpretationsspielraum zulässt.

Für ein funktionierendes Register ist auch die Aktualität der Einträge von grosser Bedeutung. Werden die

\section{Dialoggruppe Forschungsschwerpunkt Versorgungsforschung}

Versorgungsforschung ist für die Ärzteschaft ein wichtiger und wegweisender Wissenschaftsbereich. In Zeiten des Umbruchs und der Veränderungen im Gesundheitswesen (neue Finanzierungsmodelle, demographische Veränderungen, steigende Gesundheitskosten usw.) ist eine akademisch verankerte Forschung im Bereich der ärztlichen Versorgung zwingend nötig. Um wis senschaftliche, von Partikulärinteressen unabhängige Grundlagen schaffen zu können, unterstützen die Verbindung der Schweizer Ärztinnen und Ärzte (FMH) und Newlndex gemeinsam den Forschungsschwerpunkt Versorgungsforschung am ISPM Bern. Eine Dialoggruppe dient als Informations- und Austauschplattform: Vertreter der Organisationen und der Forschungsgruppen diskutieren regelmässig die laufenden und geplanten Arbeiten im Bereich der Versorgungsforschung. Die Dialoggruppe verfolgt zudem das Ziel, die Akzeptanz und Sensibilisierung innerhalb der Ärzteschaft für diesen Wissenschaftsbereich zu fördern. Die Dialoggruppe steht ihrer Basis offen für Themen-, Diskussionsvorschläge sowie für weitere Fragen und Informationen. Die Abteilung Daten, Demographie und Qualität DDQ der FMH übernimmt die Koordination der Dialoggruppe und steht für weitere Informationen und Auskünfte gerne zur Verfügung: ddq[at]fmh.ch oderTel. 0313591111

Daten erst mit mehrmonatiger Verzögerung geliefert, sind Nachfragen schwierig, aber auch Auswertungen und der Überblick über die Daten leidet.

Ein zentraler Aspekt ist schliesslich die Relevanz der gesammelten Informationen. Es ist verlockend, erst mal alle möglichen Informationen zu sammeln. Beim Aufbau eines Registers sollten aber die Ziele und der Verwendungsweck klar definiert und die zu erhebenden Daten entsprechend definiert werden.

Weiter muss der Persönlichkeitsschutz jederzeit gewährt sein. Wie werden Daten allenfalls anonymisiert, wer hat Zugriff zum Anonymisierungsschlüssel, welche Daten werden wem weitergegeben, was passiert bei einem Rückzug der Patienteneinwilligung? Die Validierung der Daten ermöglicht die Qualitätssicherung. Gesammelten Daten müssen systematisch auf fehlerhafte oder unplausible Eingaben überprüft werden, um die Qualität der Informationen möglichst hochzuhalten.

Die FMH veröffentlichte 2016 zusammen mit ANQ, H+, SAMW und Universitäre Medizin Schweiz «Empfehlungen zum Aufbau und Betrieb von gesundheitsbezogenen Registern» [8]. Die Empfehlungen münden in eine Checkliste für die Überprüfung von Registern, die 7 Dimensionen umfasst (1. Vorabklärungen bei der Planung eines Registers, 2. Konzeption des Registers, 3. Kompetenzen zur Registerführung, 4. Datenschutz und Datenhoheit, 5. Datenerhebung und Datenverwendung, 6. Qualitätssicherung, 7. Zweckänderung und Auflösung). 


\section{Der Nutzen gesammelter Daten}

Auch ein optimal geführtes Register mit vollständigen, aktuellen und relevanten Daten macht kaum Sinn, wenn die enthaltenen Informationen nicht ausgewertet und entsprechend verwendet werden.

Wie die Daten ausgewertet und verwendet werden, hängt ganz von der Bestimmung des Registers ab. Die obligatorischen Register des Bundes dienen in erster Linie als Frühwarn- und Überwachungssystem. «Zeitgerechte und wirkungsvolle Eingriffe in die Dynamik des epidemiologischen Geschehens zur Schadensabwehr und -verhütung erfordern, dass Gesundheitsgefährdungen durch übertragbare Krankheiten frühzeitig erkannt und gemeldet werden", heisst es auf der Homepage des BAG. Epidemiologische Register, die ein Gebiet oder eine Patientengruppe repräsentativ abdecken, ermöglichen auch die Identifikation möglicher Risikofaktoren. Medizinische Register, die eine bestimmte Erkrankung oder eine medizinische Behandlung abdecken, bilden häufig einzigartige Datensammlungen, die für die Weiterentwicklung der medizinischen Versorgung zentral sind.

\section{Bei optimaler Nutzung können medizinische Register Zusammenhänge aufzeigen oder Forschungsfragen anstossen.}

Da die Angaben einzelner Register begrenzt sind, ist für die Forschung eine Kombination verschiedener Register oder die Verknüpfung eines Registers mit offiziellen Angaben häufig von Interesse. Allerdings sind hier die Möglichkeiten sehr begrenzt, sei es aufgrund der technischen Machbarkeit oder aufgrund von Einschränkungen durch den Datenschutz.

Bei optimaler Nutzung können medizinische Register Zusammenhänge aufzeigen oder Forschungsfragen anstossen, sie ersetzen in der Regel aber keine randomisierten Studien. Ursache und Wirkung können in den Registern meist nicht unterschieden werden, und häufig sind die registrierten Daten nicht repräsentativ, sondern entsprechen einer bestimmten Auswahl. Dies kann etwa durch teilnehmende Kliniken, unterschiedliche Motivation bei Patienten oder geographische Unterschiede bedingt sein.

Register können aber bei der Durchführung von randomisierten Studien ebenfalls eine wichtige Rolle spielen. Bei einzelnen Erkrankungen, etwa im Bereich seltene Erkrankungen, ist es häufig schwierig, genügend mögliche Studienteilnehmerinnen und Studienteilnehmer zu identifizieren. Register können hier nützliche Kontakte liefern. Bei freiwilligen Registern muss aber auch dabei berücksichtigt werden, dass die regis- trierten Patienten nicht unbedingt repräsentativ sind. Vielleicht sind schwerer Betroffene eher registriert, vielleicht Patienten von einer bezüglich Register besonders motivierten Ärztin.

\section{Qualitätssicherung in der medizinischen Versorgung}

Im Bereich der Qualitätssicherung von implantierten Medizinprodukten («medical devices») haben freiwillige oder staatlich organisierte Register eine wichtige Rolle und führten etwa zur Erkenntnis, dass Metallauf-Metall-Hüftimplantatsysteme nicht genügend sicher sind [9-11]. Allerdings fehlt eine klare Übersicht, in welchen Ländern welche Register für implantierbare Medizinprodukte bestehen [12]. Eine solche Übersicht wird sich wohl erst mit den verstärkten Aufsichtsaufgaben der Behörden unter der angepassten Europäischen Regulierung von Medizinprodukten einstellen (https://www.swissmedic.ch/swissmedic/en/ home/medical-devices/new-eu-regulations--mdr--ivdr-. html). In der Traumatologie sollen die Informationen aus einem internationalen Traumaregister die Behandlung in Notallstationen verbessern [13]. In der Schweiz soll die gesetzlich verankerte Transplantationskohorte die Behandlungsqualität bei Transplantationen sicherstellen [14]. Hier zeigt sich auch, dass manchmal unklar ist, was als Register oder als Kohortenstudie gilt. In den USA wurde die systematische Erfassung von Transplantationen als Register bezeichnet [15]. Bei den Transplantationen ist ein klar deklariertes Ziel, die gesundheitliche Situation und weitere Behandlungen der Transplantationsempfängerinnen und -empfänger lebenslang nach der Transplantation zu dokumentieren. Dies erfordert einerseits regelmässige klinische Visiten, aber auch den geregelten Ein-

\section{Empfehlungen für den Aufbau und Betrieb von gesundheitsbezogenen Registern}

Die SAQM erarbeitete zusammen mit ANQ, $\mathrm{H}+$, SAMW und unimedsuisse Empfehlungen für den Aufbau und Betrieb von gesundheitsbezogenen Registern. Die Empfehlungen tragen zur Qualitätssicherung bei und enthalten Mindeststandards für den Aufbau und Betrieb der Register, unter anderem zu Datenschutz und Datenqualität [8]. Im Rahmen eines Piloten wurden nun zehn laufende Register anhand der Empfehlungen überprüft. Den geprüften Registern wird eine Einschätzung ihrer Aufstellung und allfälligen Optimierungsmöglichkeiten abgegeben. Gleichzeitig werden die Empfehlungen und ihre Anwendung weiterentwickelt, um die Qualität von Schweizer Registern weiter zu unterstützen.

www.saqm.ch $\rightarrow$ Publikationen $\rightarrow$ Empfehlungen Registe 
bezug von Angaben aus dem Einwohner- oder Sterberegister.

Das Schweizer Multiple Sklerose (MS) Register hingegen ist ein Beispiel für ein sogenanntes Bottom-upRegister [16]. Das Register wurde von der Schweizerischen MS Gesellschaft initiiert und wird auch von ihr

\section{Manchmal ist unklar, was als Register oder als Kohortenstudie gilt.}

finanziert. Ziel des Registers ist neben der Untersuchung von epidemiologischen Fragen primär auch die Dokumentation der Lebensqualität von Betroffenen in der Schweiz.

Im Rahmen der europaweiten Standardisierung von Health Technology Assessments (THA) wurde kürzlich erhoben, in welcher Form und unter welchen Voraussetzungen Informationen aus Registern von verschiedenen HTA-Behörden miteinbezogen werden [17]. Dabei stellte sich heraus, dass Registerdaten öfter verwendet werden als erwartet, aber auch dass Register für den Einbezug in HTA in mehreren Dimensionen Qualitätskriterien erfüllen müssen. Basierend auf dieser Untersuchung soll nun ein QualitätsassessmentTool entwickelt werden.

\section{Ausblick}

Register, die als ein Zweig von «Big Data» betrachtet werden können, gewinnen voraussichtlich in den kommenden Jahren weiter an Bedeutung. Sie sind aber keine Zauberwaffe, sondern bedürfen eines überlegten Aufbaus sowie grösster Sorgfalt, damit sie einen Beitrag zur Forschung und Qualitätssicherung leisten

\section{Es sollte angestrebt werden, eine einheitliche nicht-sprechende Identifikationsnummer in den verschiedenen Registern und Routinedaten zu verwenden.}

können. Wie generell bei Big Data gilt auch bei den Registern: Wenn Schrott eingegeben wird, wird auch Schrott herauskommen. Die Verknüpfbarkeit und eine Harmonisierung der Daten zwischen Registern und Routinedaten ist entscheidend für den Ausbau und die Zukunft der Nutzung von Registern im Gesundheitswesen. Das Netzwerk Swiss Personalized Health Network (SPHN) fördert seit 2017 Projekte, welche die Voraussetzungen verbessern und schaffen, «um den für die Forschung notwendigen Austausch von gesund- heitsbezogenen Daten zu ermöglichen» (https://www. sphn.ch). Zudem sollte angestrebt werden, eine einheitliche nicht-sprechende Identifikationsnummer in den verschiedenen Registern und Routinedaten zu verwenden. Hier sind noch rechtliche Aspekte und solche des Datenschutzes zu klären. Es ist zu hoffen, dass das NFP 74 (Nationales Forschungsprogramm "Gesundheitsversorgung", http://www.nfp74.ch) und andere Programme zur Stärkung der Versorgungsforschung genügend Anlass bieten, hier Fortschritte zu erzielen

Literatur

1 Hostettler S, Hersperger M. Abschluss des Projekts «Forum medizinische Register Schweiz»: Medizinische Register in der Schweiz auf einen Blick. Schweizerische Ärzteztg. 2012;93:991-2.

2 Weddell JM. Registers and registries: a review. Int J Epidemiol. 1973;2(3):221-8.

3 Alter M. Medical registers. Advances in neurology. 1978;19:121-39.

4 Zaletel M, Kralj M. Methodological guidelines and recommendations for efficient and rational governance of patient registries. Ljubljana: National Institute of Public Health; 2015.

5 Mathis-Edenhofer S, Piso B. Types of medical registries - definitions, methodological aspects and quality of the scientific work with registries. Wien Med Wochenschr. 2011;161(23-24):580-90.

6 Mathis S, Wild C. Register für klinische und gesundheitsökonomische Fragestellungen: Einsatzbereiche von kardiovaskulären, wirbelsäulenspezifischen und neurologischen Registern und Good Practice Strategien für die Arbeit mit Registern. HTA Projektbericht \#11, 2008.

7 Hostettler S, Hersperger M, Herren D. Medizinische Register: Wo liegt der Schlüssel zum Erfolg? Schweizerische Ärzteztg. 2012;93:1251-5.

$8 \mathrm{ANQ}, \mathrm{FMH}, \mathrm{H}+$, et al. Empfehlungen zum Aufbau und Betrieb von gesundheitsbezogenen Registern. Bern; 2016

9 Smith AJ, Dieppe P, Howard PW, et al. Failure rates of metal-onmetal hip resurfacings: analysis of data from the National Joint Registry for England and Wales. Lancet. 2012;380(9855):1759-66.

10 Sedrakyan A. Metal-on-metal failures - in science, regulation, and policy. Lancet. 2012;379(9822):1174-6

11 Sedrakyan A, Paxton E, Graves S, et al. National and international postmarket research and surveillance implementation: achievements of the International Consortium of Orthopaedic Registries initiative. The Journal of bone and joint surgery American volume. 2014;96(Suppl 1):1-6.

12 Niederlander C, Wahlster P, Kriza C, et al. Registries of implantable medical devices in Europe. Health Policy. 2013;113(1-2):20-37.

13 Hartel MJ, Jordi N, Evangelopoulos DS, et al. Optimising care in a Swiss University Emergency Department by implementing a multicentre trauma register (TARN): report on evaluation, costs and benefits of trauma registries. Emerg Med J. 2011;28(3):221-4.

14 Koller MT, van Delden C, Müller NJ, et al. Design and methodology of the Swiss Transplant Cohort Study (STCS): a comprehensive prospective nationwide long-term follow-up cohort. European Journal of Epidemiology. 2013;28(4):347-55.

15 Organ Procurement and Transplantation Network and Scientific Registry of Transplant Recipients 2010 Data Report. American Journal of Transplantation. 2012;12(s1):1-156.

16 Steinemann N, Kuhle J, Calabrese P, et al. The Swiss Multiple Sclerosis Registry (SMSR): study protocol of a participatory, nationwide registry to promote epidemiological and patient-centered MS research. BMC Neurology. 2018;18(1):111.

17 Mandeville KL, Valentic M, Ivankovic D, et al. Quality assurance of registries for health technology assessment. International Journal of Technology Assessment in Health Care. 2018;34(4):360-7. 